\title{
Coqa: Concurrent Objects with Quantized Atomicity
}

\author{
Yu David Liu, Xiaoqi Lu, and Scott F. Smith \\ Department of Computer Science \\ The Johns Hopkins University \\ $\{y l i u, x i a o q i l u$, scott $\} @ c s . j h u . e d u$
}

\begin{abstract}
This paper introduces a new language model, Coqa, for deeply embedding concurrent programming into objects. Every program written in our language has the desirable behaviors of atomicity, mutual exclusion, and race freedom automatically built in. A key property of our model is the notion of quantized atomicity: every concurrent program execution can be viewed as being divided into quantum regions of atomic execution, greatly reducing the number of interleavings to consider. Rather than building atomicity locally, i.e. declaring some code blocks as atomic blocks and leaving other code segments with no guarantee of any atomicity property, we build it in globally, so that a form of atomicity, quantized atomicity, ubiquitously exists at all program points. We justify our approach both from a theoretical basis by showing that a formal representation, KernelCoqa, has provable quantized atomicity properties, and by implementing CoqaJava, a Java extension incorporating all of the Coqa features.
\end{abstract}

\section{Introduction}

Coqa (for Concurrent objects with quantized atomicity) is a new object-oriented language aimed at facilitating programming in a multi-core CPU environment. Programming multi-core CPUs requires much greater programmer skill, and is one of the most significant new demands programmers will face in the coming decade. The design goal of Coqa is to build a language in which it is easier to naturally write concurrent programs with good concurrency properties. Unlike Java where good properties such as race freedom can only be achieved if the programmer explicitly declares it by using synchronized, the "default" mode in Coqa is inverted: good properties of race freedom, mutual exclusion, and atomicity are preserved unless programmers explicitly declare otherwise.

Existing concurrent object language designs are numerous and include for example Agh90 Arm96 MilBST00. What makes our work novel is the intrinsic properties Coqa preserves. Most important is atomicity, i.e. the property that a block of code can always be viewed as occurring atomically no matter what interleaving it is involved in. With tightly coupled computation running on multi-core CPUs, data sharing between threads is very common and the patterns are more complex than on a single-core CPU due to random variations in scheduling. To support atomicity, Coqa takes the route of "atomicity-by-design" for each

L. Hendren (Ed.): CC 2008, LNCS 4959, pp. 260-275, 2008.

(C) Springer-Verlag Berlin Heidelberg 2008 
method: atomicity is ubiquitous because by default each complete method execution is observably atomic. Note this is much stronger than the synchronized methods of Java: the Coqa method and all methods it invokes are viewed as happening atomically. The synchronized methods in Java only provide a shallow notion of mutual exclusion.

One particular challenge of whole-method atomicity is that it can be overly strong, and the resulting executions will not be efficient, or may even deadlock if there is significant contention across methods. For this reason, Coqa allows programmers to relax whole-method atomicity by dividing a method into a small number of discrete zones of atomicity (called quanta in Coqa), and each quantum is serializable regardless of the interleaving of the actual execution. This property, called quantized atomicity, is preserved for all Coqa programs. The main appeal is to significantly reduce the number of interleavings possible in concurrent program runs, and thus to ease the debugging burden. If two pieces of code each have 100 execution steps, reasoning tools would have to consider $C_{200}^{100}$ (i.e., around $10^{58}$ ) interleaving scenarios; however, if the aforementioned 100 steps can be split into 3 atomic quanta, there are only $C_{6}^{3}=20$ possibilities to consider. With quantized atomicity, next-generation verification tools can potentially enumerate all interleaving scenarios, a strategy largely impractical today. Actors Agh90 AMST97] were in some sense the starting point for the design of Coqa: atomicity is preserved for each Actor method because its execution once initiated does not depend on the state of other actors and each method is therefore trivially serializable. Actors' ubiquitous atomicity arises from the fact that the model supports only asynchronous messaging, and so methods once initiated cannot receive outside inputs.

Another design goal of Coqa is to make a concurrent language design that naturally meshes well with object-oriented language features. This stands in contrast to the non-object-based syntax and semantics commonly used in existing languages for concurrent programming. Language abstractions such as library class Thread, thread spawning via its start method and synchronized blocks in Java, and the atomic blocks in various Software Transactional Memory (STM) systems that have been adopted into OO languages $\mathrm{CMC}^{+} 06$, are not that different from what was used three decades ago in non-object-oriented languages [Lom77.

Existing language models fall short of achieving the goals of both ubiquitous atomicity and easy OO-style concurrent programming. Ubiquitous atomicity is a global property of all programs; Java does not have a notion of atomicity built into the language and the form of atomicity in STM systems is only local atomicity. STM systems also require rollbacks to deal with atomicity-breaking contentions and are known to be inapplicable to I/O-intensive applications, such as GUI and network systems, so they can never be ubiquitous. Out of the desire of pervasiveness, we take a blocking and not a rollback approach to achieve atomicity. The Actor model achieves ubiquitous atomicity, but programming in Actors is very different from what programmers are used to, since with pure asynchronous messaging any processing of a message reply must be handled by 


\begin{tabular}{|c||c|c|}
\hline messaging & what it is & why you should use it \\
\hline \hline$\circ \cdot \mathrm{m}(\mathrm{v})$ & intra-task messaging & promotes mutual exclusion and atomicity \\
\hline$\circ \rightarrow \mathrm{m}(\mathrm{v})$ & task creation & promotes parallelism by starting up a new task \\
\hline$\circ \Rightarrow \mathrm{m}(\mathrm{v})$ & sub-tasking & promotes parallelism by encouraging early free \\
\hline
\end{tabular}

Fig. 1. The Three Messaging Mechanisms and Their Relative Strengths

a completely new message, necessarily chopping up methods into many small pieces. So, Coqa shares the spirit of ubiquitous atomicity of Actors, but allows more familiar synchronous messaging syntax to be used which avoids the need to break up methods.

In this paper, we formalize Coqa in a formal system called KernelCoqa, in which we prove the properties of quantized atomicity, mutual exclusion and race freedom. We have also implemented a prototype language CoqaJava as a Java extension which simply replaces Java threads with our new forms of object messaging.

\section{Informal Overview}

The concurrency unit in our language is a task. A task is a unit of execution that can potentially be interleaved with other units. Tasks are closely related to (logical) threads, but come with inherent atomicity properties not found in threads, and we coin a new term to reflect this distinction. Coqa has a very simple syntax: the only difference from the Java object model is a richer syntax to support object messaging, as summarized in Fig. 1. Beyond the familiar $\circ . \mathrm{m}(\mathrm{v})$ message sending expression, $\circ \rightarrow \mathrm{m}(\mathrm{v})$ and $\circ \Rightarrow \mathrm{m}(\mathrm{v})$ are additionally provided for task creation (a form of thread spawning) and subtasking (a form of thread open nesting), respectively.

The Running Example. Throughout the section, we will use a simple example of basic banking operations, including account opening and balance transfer operations, as shown in Fig. 2. Bank accounts are stored in a hash table, implemented in a standard manner with bucket lists.

\subsection{Task Creation}

Tasks are created by simply sending asynchronous messages to objects, using the $\circ \rightarrow \mathrm{m}(\mathrm{v})$ expression. This is a more "object-based" thread creation than the current practice in Java, where a special Thread class is used. This notion is more aligned with Actor languages, where all message passings can be viewed as thread creations. In Fig. 2, the top-level main method starts up three concurrent tasks, two balance transfers and one account opening, by the invocations of lines M1, M2 and M3. Syntax bk $\rightarrow$ transfer ("Alice", "Bob", 3) indicates an asynchronous 


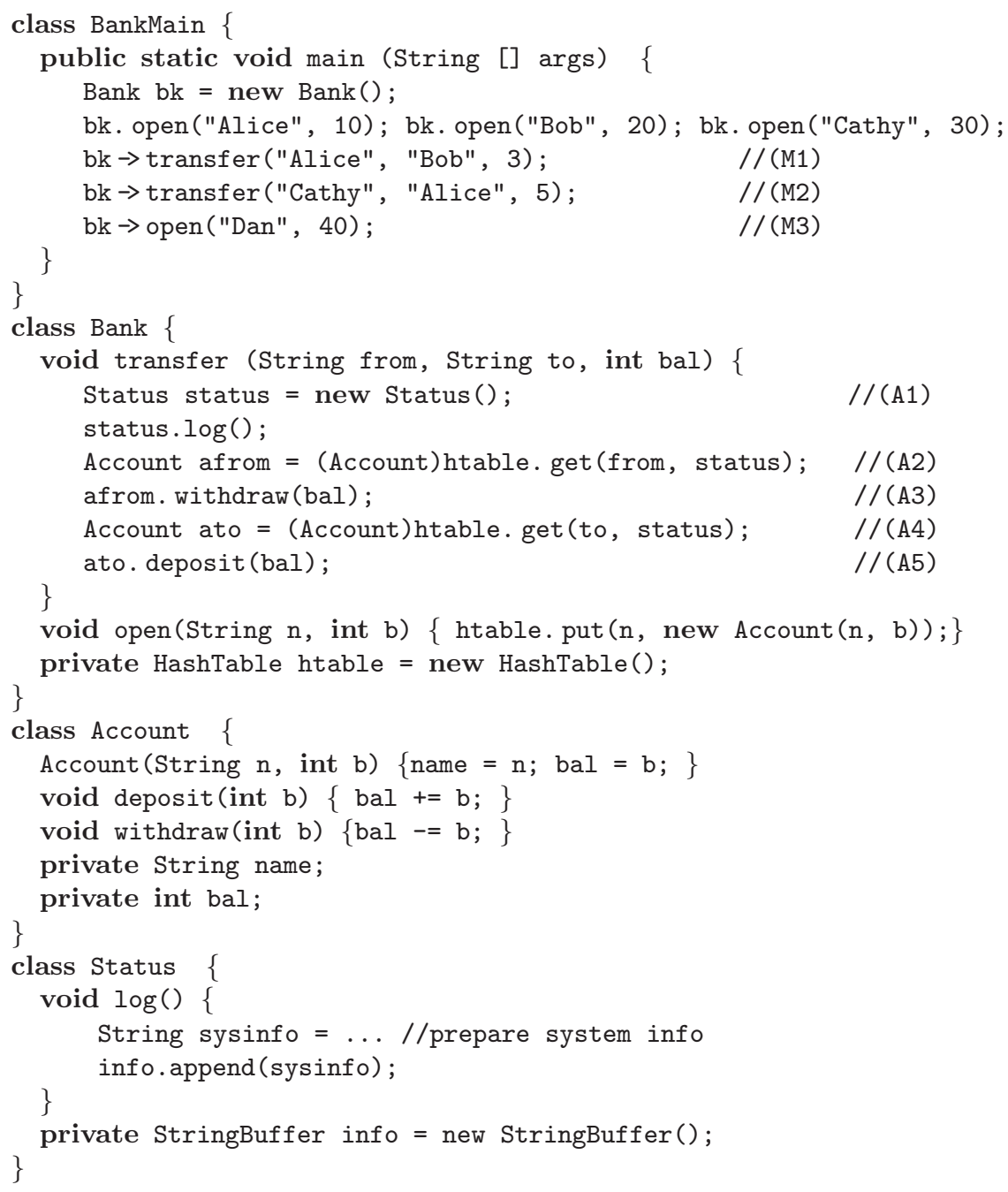

Fig. 2. A Banking Program

message transfer sent to object bk with indicated arguments. Asynchronous message sending returns immediately, so the sender can continue, and a new task is created to execute the invoked method. This new task terminates when its method is finished. To keep the language simple, asynchronous invocations in Coqa do not return values. 


\section{$2.2 \quad$ Intra-task Messaging}

Message sending $\circ . \mathrm{m}(\mathrm{v})$ is the same syntax as Java, but has different semantics giving stronger atomicity properties: when invoked, object $\mathrm{o}$ will be captured by the invoking task and cannot be used by other tasks until the current task is complete. Capturing is a blocking mechanism, but unlike Java where programmers need to explicitly specify what to lock and when to lock, the capture and blocking of objects is built into Coqa.

This intuitive definition for $\circ . \mathrm{m}(\mathrm{v})$ is the programmer view, but is not an efficient implementation strategy: only mutation affects the preservation of atomicity, and so we actually only need to capture objects "lazily" when their fields are read and written. Our notion of "capture" is a standard two-phase non-exclusive read lock and exclusive write lock Gra78. When an object's field is read, the object is said to be read captured; when the field is written, the object is said to be write captured. The same object can be read captured by multiple tasks at the same time, but to be write captured, the object has to be exclusively owned, i.e. not read captured or write captured by another task. Two-phase locking optimizes our model since reads are overwhelmingly more common than writes in most programs. Many other optimizations are also possible by static analysis, a topic we leave to future work.

The preservation of atomicity can be seen in the invocation of the transfer method of Fig. 2. the HashTable object referenced by htable is captured by a task, say the task created in line M1, and will not be released until the end of the method (and hence, the task). Therefore it is not possible for one transfer task to be reading from the HashTable object while at the same time a different transfer task is writing to it.

\section{$2.3 \quad$ Subtasking}

The model we have presented thus far admits significant parallelism if most object accesses are read accesses. Blocking is possible, however, when frequent writes are needed. For instance, consider the parallel execution of the two tasks spawned by (M1) and (M3). One of them will be blocked as (M1) reads from the HashTable object, while (M3) attempts to write 1 And the task being blocked cannot make any progress until the other task completes and releases its captured object. Intuitively, the task of adding Dan as a new account, (M3), is totally unrelated to the task of transferring money from Alice to Bob, (M1), except for their shared access to the HashTable object. There should be at least some parallelism possible between the two tasks.

Coqa achieves this by allowing programmers to spawn off the access of the HashTable object (and all objects it indirectly accesses) as a new subtask. The high-level meaning behind a subtask is that it achieves a relatively independent goal; its completion signals a partial victory so that the captured objects used

\footnotetext{
${ }^{1}$ Strictly speaking, the read-write conflict happens on the object representing the bucket list inside the HashTable, but we omit this detail since we do not have space to give the source code for the internals of the HashTable.
} 
to achieve this subtask can be "freed", i.e. no longer considered captured. In terms of syntax, the only change to the source code of transfer in Fig. 2 is to change the dot (.) messagings at (A2) and (A4) to $\Rightarrow$ for subtask creation messaging. In this case, the task $t$ created at (M1) spawns a subtask $t^{\prime}$ at (A2) via $\Rightarrow$. The HashTable object will be captured by $t^{\prime}$ but not $t$. More parallelism is achieved by such subtasking: other tasks waiting to capture the HashTable object would have to block for the duration of $t$ instead of the much shorter span of $t^{\prime}$ if (.) was used. Subtasking is a synchronous invocation, i.e., the task executing transfer waits until its subtask executing get returns a result. But the subtask has a distinct capture set of its own. And like a task, a subtask frees objects in its capture set when it finishes.

A subtask is also a task, so it prevents arbitrary interleaving. The change in line (A2) from (.) to $\Rightarrow$ admits interleaving between task (M1) and (M3) that was not allowed before, but it does not mean that arbitrary interleaving can occur; for example, if M1 were in the middle of a key lookup M3 still cannot add a new bucket. We will discuss such concurrency properties in the presence of subtasking later in this section.

Subtasking is related to open nesting in STM systems $\mathrm{NMAT}^{+} 07 \mathrm{CMC}^{+} 06$. Open nesting is used to nest a transaction inside another transaction, where the nested transaction can commit before the enclosing transaction runs to completion. While the mechanism of open nesting of transactions can be summarized as early commit, subtasking can be summarized as early release.

Capture set inheritance. One contentious issue for open nesting is the case where a nested transaction and the transactions enclosing it both need the same object. For instance in Atomos $\left[\mathrm{CMC}^{+} 06\right.$, the issue is circumvented by restricting the $\mathrm{read} /$ write sets to be disjoint between the main and nested transaction. When the same issue manifests itself in the scenario of subtasking, the question is, "Can a subtask access objects already captured by its enclosing task(s)?"

We could in theory follow Atomos' approach. This however would significantly reduce programmability. Let us consider the example of the Status object in the transfer method. From the programmer's view, this object keeps track of the system status throughout the execution of the transfer method. However, if the Atomos's approach were taken, a subtask spawned by the transfer task for accessing the HashTable would not be able access the Status object because this object has already been captured by the transfer task. Even worse, a deadlock would be introduced in this case.

We believe the essence of having a subtasking relationship between a parent and a child is that the parent should generously share its resources with the child. Therefore accessing the Status in the subtask is perfectly legal in Coqa. Observe that the relationship between a task and its subtask is synchronous, so there is no concern of interleaving between a task and its subtask.

\subsection{Properties}

Quantized Atomicity Some tasks simply should not be considered wholly atomic because they are fundamentally needing to share data with other tasks, and 
for this case it is simply impossible to have full atomicity over the whole task. The main reason why a programmer wants to declare a subtask is to open a communication channel with other tasks for such sharing, as was illustrated in the subtasking example above. With subtasking, objects captured by the subtask can serve as communication points between different tasks. This is because the objects freed at the end of one subtask might be recaptured later, and the object may have been mutated by the original subtask.

Quantized atomicity is the property that for any task, its execution sequence can be viewed as a sequence of atomic regions, the atomic quanta, demarcated by task and subtask creation points. This atomicity property is weaker than a whole task being atomic, but as long as full task atomicity is broken only when it is really necessary (that is, a minimal number of $\Rightarrow$ and $\rightarrow$ messagings are used), the atomic quanta will each be large, and significant reduction of interleaving can be achieved. In reality, what matters is not that the entire method must be atomic, but that the method admits a drastically limited number of interleaving scenarios. Quantized atomicity aims to strikes a balance between what is realistic and what is reasonable.

Mutual Exclusion. For objects accessed by synchronous messaging, the property of mutual exclusion over mutation spans the lifetime of the current task, even across the boundaries of quanta. For instance, over the entire duration of any task executing transfer in Fig. 2, the Status object is guaranteed not to be mutated by any other task before the current transfer ends, even if other tasks have reference to Status. Our notion of object mutual exclusion is much stronger than what Java's synchronized provides: Java only guarantees the object with the method is itself not mutated by other threads, while we are guaranteeing the property for all objects which are directly or indirectly sent synchronous messages to at run time by the method, many of which may be unknown to the caller.

Race Freedom. In Coqa, we show that two tasks cannot race to access any object field, except in the case where both may only read from the same object field.

A Simple Memory Model. With these concurrency properties, Coqa eliminates the need for the overly complex memory model of Java [MPA05.

\section{Formalization}

In this section we present KernelCoqa, a small formal kernel language of Coqa.

We first define some basic notation used in our formalization. We write $\overline{x_{n}}$ as shorthand for a set $\left\{x_{1}, \ldots, x_{n}\right\}$, with $\emptyset$ as empty set. $\overrightarrow{x_{n} \mapsto y_{n}}$ denotes a mapping $\left\{x_{1} \mapsto y_{1}, \ldots, x_{n} \mapsto y_{n}\right\}$, where $\left\{x_{1}, \ldots x_{n}\right\}$ is the domain of the mapping, $\operatorname{dom}(H)$. We also write $H\left(x_{1}\right)=y_{1}, \ldots, H\left(x_{n}\right)=y_{n}$. When no confusion arises, we drop the subscript $n$ for sets and mapping sequences. We write $H\{x \mapsto y\}$ as a mapping update: if $x \in \operatorname{dom}(H), H$ and $H\{x \mapsto y\}$ are identical except that 


\begin{tabular}{|c|c|c|c|}
\hline & & $H \quad::=$ & $\overrightarrow{o \mapsto\langle c n ; R ; W ; F\rangle}$ \\
\hline$P::=$ & $\overrightarrow{c n \mapsto\langle l ; F d ; M d\rangle}$ & $::=$ & $\overrightarrow{f n \mapsto v}$ \\
\hline$F d::=$ & $\overline{f n}$ & $T \quad::=$ & $\langle t ; \gamma ; e\rangle \mid T \| T^{\prime}$ \\
\hline$M d::=$ & $\overrightarrow{m n \mapsto \lambda x . e}$ & $N \quad::=$ & $\overrightarrow{t \mapsto t^{\prime}}$ \\
\hline$e \quad::=$ & null $|x|$ cst $\mid$ this & $R, W::=$ & $\bar{t}$ \\
\hline & new $c n$ & $\gamma \quad::=$ & $o$ | null \\
\hline & $f n \mid f n=e$ & $v \quad::=$ & cst | o | null \\
\hline & e.mn $(e)$ & $e \quad::=$ & $v \mid$ wait $t$ \\
\hline & $e \rightarrow m n(e)$ & | & $e \uparrow e \mid \ldots$ \\
\hline & $e \Rightarrow m n(e)$ & $\mathbf{E} \quad::=$ & - $f n=\mathbf{E}$ \\
\hline & let $x=e$ in $e$ & & E. $m(e) \mid v \cdot m(\mathbf{E})$ \\
\hline$l \quad::=$ & exclusive $\mid \epsilon$ & & $\mathbf{E} \rightarrow m(e) \mid v \rightarrow m(\mathbf{E})$ \\
\hline cst & constant & & $\mathbf{E} \Rightarrow m(e) \mid v \Rightarrow m(\mathbf{E})$ \\
\hline$c n$ & class name & & let $x=\mathbf{E}$ in $e$ \\
\hline$m n$ & method name & $O$ & object ID \\
\hline$f n$ & field name & $t$ & task $I D$ \\
\hline$x$ & variable name & $\operatorname{anc}(N, t)$ & $=\left\{\begin{array}{l}\{t\}, \text { if } N(t)=\text { null } \\
\{t\} \cup \operatorname{anc}\left(N, t^{\prime}\right), \text { if } N(t)=t^{\prime}\end{array}\right.$ \\
\hline
\end{tabular}

Fig. 3. Language Abstract Syntax and Dynamic Data Structure

$H\{x \mapsto y\}$ maps $x$ to $y$; if $x \notin \operatorname{dom}(H), H\{x \mapsto y\}=H, x \mapsto y . H \backslash x$ removes the mapping $x \mapsto H(x)$ from $H$ if $x \in \operatorname{dom}(H)$, otherwise the operation has no effect.

KernelCoqa is an idealized object-based language with objects, messaging, and fields. Its abstract syntax is shown on the left of Fig. 3. A program $P$ is a set of classes. Each class has a unique name $c n$ and its definition consists of sequences of field $(F d)$ and method $(M d)$ declarations. To make the formalization feasible, many features are left out, including types and constructors. Besides local method invocations via dot (.) notation, synchronous and asynchronous messages are sent to objects using $\Rightarrow$ and $\rightarrow$, respectively. A class declared exclusive will have its objects write captured upon any access. This label is useful for eliminating deadlocks inherent in a two-phase locking strategy, such as when two tasks first read capture an object, then both try to write capture the same object and thus deadlock.

Operational Semantics. Our operational semantics is defined as a contextual rewriting system over states $S \Rightarrow S$, where each state is a triple $S=(H, N, T)$ for $H$ the object heap, $N$ a task ancestry mapping, and $T$ a set of parallel tasks. Every task has a local evaluation context $\mathbf{E}$. The relevant definitions are given in Fig. 3. $H$ is a mapping from objects $o$ to field records tagged with their class name $c n$. In addition, each $o$ has capture sets, $R$ and $W$, for recording tasks that have read or write captured this object. A task is a triple consisting of the task ID $t$, the object $\gamma$ this task currently operates on, and an expression $e$ to be evaluated. 
SET

$$
\begin{gathered}
H(\gamma)=\langle c n ; R ; W ; F\rangle \\
\frac{H^{\prime}=H\{\gamma \mapsto\langle c n ; R ; W \cup\{t\} ; F\{f n \mapsto v\}\rangle\} \text { if } R \subseteq \operatorname{anc}(N, t), W \subseteq \operatorname{anc}(N, t)}{H, N,\langle t ; \gamma ; \mathbf{E}[f n=v]\rangle \Rightarrow H^{\prime}, N,\langle t ; \gamma ; \mathbf{E}[v]\rangle}
\end{gathered}
$$

GeT

$$
\begin{gathered}
H(\gamma)=\langle c n ; R ; W ; F\rangle \quad P(c n)=\langle l ; M d ; F d\rangle \quad F(f n)=v \\
H^{\prime}=\left\{\begin{array}{c}
H\{\gamma \mapsto\langle c n ; R ; W \cup\{t\} ; F\rangle\}, \text { if } l=\operatorname{exclusive,} R \subseteq \operatorname{anc}(N, t), W \subseteq \operatorname{anc}(N, t) \\
H\{\gamma \mapsto\langle c n ; R \cup\{t\} ; W ; F\rangle\}, \text { if } l=\epsilon, W \subseteq \operatorname{anc}(N, t)
\end{array}\right. \\
H, N,\langle t ; \gamma ; \mathbf{E}[f n]\rangle \Rightarrow H, N,\langle t ; \gamma ; \mathbf{E}[v]\rangle
\end{gathered}
$$

INVOKE

$$
\frac{H(o)=\langle c n ; R ; W ; F\rangle \quad P(c n)=\langle l ; F d ; M d\rangle \quad M d(m n)=\lambda x . e}{H, N,\langle t ; \gamma ; \mathbf{E}[o . m n(v)]\rangle \Rightarrow H, N,\langle t ; o ; \mathbf{E}[e\{v / x\} \uparrow \gamma]\rangle}
$$

$\operatorname{TASK}\left(t, \gamma, m n, v, o, t^{\prime}\right)$

$$
\frac{t^{\prime} \text { fresh }}{H, N,\langle t ; \gamma ; \mathbf{E}[o \rightarrow m n(v)]\rangle \Rightarrow H, N,\langle t ; \gamma ; \mathbf{E}[\text { null }]\rangle \|\left\langle t^{\prime} ; o ; \operatorname{this.} m n(v)\right\rangle}
$$

$\operatorname{SuBTASK}\left(t, \gamma, m n, v, \gamma, t^{\prime}\right)$

$$
\frac{N^{\prime}=N\left\{t^{\prime} \mapsto t\right\} \quad t^{\prime} \text { fresh }}{H, N,\langle t ; \gamma ; \mathbf{E}[o \Rightarrow m n(v)]\rangle \Rightarrow H, N^{\prime},\left\langle t ; \gamma ; \mathbf{E}\left[\text { wait } t^{\prime}\right]\right\rangle \|\left\langle t^{\prime} ; o ; \text { this.mn }(v)\right\rangle}
$$

$$
\frac{H^{\prime}=\biguplus_{H(o)=\langle c n ; R ; W ; F\rangle}(o \mapsto\langle c n ; R \backslash t ; W \backslash t ; F\rangle) \quad N(t)=\text { null }}{H, N,\langle t ; \gamma ; v\rangle \Rightarrow H^{\prime}, N, \epsilon}
$$

$$
\begin{aligned}
& \operatorname{STEnd}\left(t, v, t^{\prime}\right) \\
& \frac{H^{\prime}=\biguplus_{H(o)=\langle c n ; R ; W ; F\rangle}(o \mapsto\langle c n ; R \backslash t ; W \backslash t ; F\rangle) \quad N(t)=t^{\prime}}{H, N,\langle t ; \gamma ; v\rangle \|\left\langle t^{\prime} ; \gamma^{\prime} ; \mathbf{E}[\text { wait } t]\right\rangle \Rightarrow H^{\prime}, N \backslash t,\left\langle t^{\prime} ; \gamma^{\prime} ; \mathbf{E}[v]\right\rangle}
\end{aligned}
$$

Fig. 4. KernelCoqa Core Operational Semantics Rules

The core single-step evaluation rules are presented in Fig. 4. The rules for LET, RETURN and other standard constructs are omitted here; see [Lu07]. The rules implicitly operate over some fixed program $P$. The Invoke rule for intratask messaging is interpreted as a standard function application. The TAsk rule creates a new task via asynchronous messaging. The SUBTASK rule creates a subtask of the current task via synchronous messaging, and the parent task enters a wait state until the subtask returns. When a task finishes, all objects it has captured are freed; the TEND and STEND are rules for ending a task and a subtask, respectively. The two-phase locking capture policy is implemented in 
the SET and the GET rules. The optional exclusive modifier requires an object to be write captured in both rules. When a task cannot capture an object it needs, it is implicitly object-blocked on the object until it is entitled to capture it - the SET/GET rule cannot progress.

Atomicity Theorems. Here we formally establish the informal claims about KernelCoqa: quantized atomicity, mutual exclusion of tasks, and race freedom. Proofs are provided in Lu07. The key Lemma is the Bubble-Down Lemma, Lemma 1, which shows that consecutive steps of a certain form in a computation path can be swapped to give an equivalent path. Then, by a series of bubblings, each quantum of steps can be bubbled to all be consecutive in an equivalent computation path, showing that the quanta are serializable: Theorem [1. The technical notion of a quantum is the pmsp below, a pointed maximal subpath. These are a series of local steps of one task with a nonlocal step at the end, which may be embedded in a larger concurrent computation path. We prove in Theorem 1 that any computation path can be viewed as a collection of pmsp's, and all pmsp's in the path are serializable and thus the whole path is.

Definition 1 (Object State). Recall the global state is a triple $S=(H, N, T)$. The object state for $o$, written $s_{o}$, is defined as $H(o)$, the value of the object $o$ in the current heap $H$, or null if $o \notin \operatorname{dom}(H)$.

Definition 2 (Local and Nonlocal Step). A step st $t_{r}=\left(S, r, S^{\prime}\right)$ denotes a transition $S \Rightarrow S^{\prime}$ by rule $r$ of Figure 4. st $t_{r}$ is a local step if $r$ is one of the local rules: either GET, SET, ThIS, LET, RETURN, Inst or Invoke. st $t_{r}$ is a nonlocal step if $r$ is one of nonlocal rules: either TASK, SUBTASK, TEND or STEND.

Every nonlocal rule has a label given in Fig 4 used as the observable.

Definition 3 (Computation Path). A computation path $p$ is a finite sequence of steps st $t_{r_{1}} \ldots s t_{r_{i}}$ such that $s t_{r_{1}} s t_{r_{2}} \ldots s t_{r_{i-1}} s t_{r_{i}}=\left(S_{0}, r_{1}, S_{1}\right)\left(S_{1}, r_{2}, S_{2}\right)$ $\ldots\left(S_{i-2}, r_{i-1}, S_{i-1}\right)\left(S_{i-1}, r_{i}, S_{i}\right)$.

When no confusion arises, we simply call it a path.

Definition 4 (Observable Behavior). The observable behavior of a path $p$, $o b(p)$, is the sequence of labels for the nonlocal steps in $p$.

Note that this definition encompasses I/O behavior elegantly since I/O in KernelCoqa can be viewed as a fixed object which is sent nonlocal and thus observable messages.

Definition 5 (Observable Equivalence). Two paths $p_{1}$ and $p_{2}$ are observably equivalent, written $p_{1} \equiv p_{2}$, iff $o b\left(p_{1}\right)=o b\left(p_{2}\right)$.

Definition 6 (Object-blocked). A task $t$ is in an object-blocked state $S$ at some point in a path $p$ if it would be enabled for a next step st $t_{r}=\left(S, r, S^{\prime}\right)$ for which $r$ is a GET or SET step on object o, except for the fact that there is a capture violation on : one of preconditions of the GET/SET fails to hold in $S$ and so st cannot in fact be the next step at that point. 
Definition 7 (Sub-path and Maximal Sub-path). Given a path p, for some $t$ a sub-path $s p_{t}$ of $p$ is a sequence of steps in $p$ which are all local steps of task t. A maximal sub-path is a $s p_{t}$ in $p$ which is longest: no local $t$ steps in $p$ can be added to the beginning or the end of $s p_{t}$ to obtain a longer sub-path.

Definition 8 (Pointed Maximal Sub-path). For a given path, a pointed maximal sub-path for $t$ ( mmsp $\left._{t}\right)$ is a maximal sub-path $s p_{t}$ with either 1 ) it has one nonlocal step appended to its end or 2) there are no more $t$ steps ever in the path.

The second case is the technical case of when the (finite) path has ended but the task $t$ is still running. The last step of a $p m s p_{t}$ is called its point.

The $p m s p$ 's are the units which we need to serialize: they are all spread out in the initial path $p$, and we need to show there is an equivalent path where each pmsp runs in turn as an atomic unit.

Definition 9 (Task Indexed pmsp). For some fixed path $p$, define pmsp $_{t, i}$ to be the $i^{\text {th }}$ pointed maximal sub-path of task $t$ in $p$, where all the steps of the pmsp $p_{t, i}$ occur after any of $p m s p_{t, i+1}$ and before any of $p m s p_{t, i-1}$.

Definition 10 (Waits-for and Deadlocking Path). For some path $p$, pmsp $p_{t_{1}, i}$ waits-for pmsp $_{t_{2}, j}$ if $t_{1}$ goes into a object-blocked state in pmsp $_{t_{1}, i}$ on an object captured by $t_{2}$ in the blocked state. A deadlocking path $p$ is a path where this waits-for relation has a cycle: $p m s p_{t_{1}, i}$ waits-for $p m s p_{t_{2}, j}$ while $p m s p_{t_{2}, i^{\prime}}$ waits-for $p m s p_{t_{1}, j^{\prime}}$.

From now on we assume in this theoretical development that there are no such cycles. In Coqa deadlock is an error that should have not been programmed to begin with, and so deadlocking programs are not ones we want to prove facts about.

Definition 11 (Quantized Sub-path and Quantized Path). A quantized sub-path contained in $p$ is a pmsp $p_{t}$ of $p$ where all steps of pmsp $_{t}$ are consecutive in $p$. A quantized path $p$ is a path consisting of a sequence of quantized sub-paths.

The main technical Lemma is the following Bubble-Down Lemma, which shows how local steps can be pushed down in a path. Use of such a Lemma is the standard technique to show atomicity properties. Lipton Lip75 first described such a theory, called reduction; his theory was later refined by [LS89].

Definition 12 (Equivalent Step Swap). For two consecutive steps st $t_{r_{1}} s t_{r_{2}}$ in a path $p$, where $s t_{r_{1}} \in$ pmsp $_{t_{1}}$, st $t_{r_{2}} \in$ pmsp $_{t_{2}}, t_{1} \neq t_{2}$ and $s t_{r_{1}} s t_{r_{2}}=$ $\left(S, r_{1}, S^{\prime}\right)\left(S^{\prime}, r_{2}, S^{\prime \prime}\right)$, if the step swap of $s t_{r_{1}} s t_{r_{2}}$, written as $s t_{r_{2}}^{\prime}$ st $t_{r_{1}}^{\prime}$, gives a new path $p^{\prime}$ such that $p \equiv p^{\prime}$ and $s t_{r_{2}}^{\prime} s t_{r_{1}}^{\prime}=\left(S, r_{2}, S^{*}\right)\left(S^{*}, r_{1}, S^{\prime \prime}\right)$, then it is an equivalent step swap.

Lemma 1 (Bubble-down Lemma). For any path $p$ with any two consecutive steps $s t_{r_{1}} s t_{r_{2}}$ where $s t_{r_{1}} \in$ wmsp $_{t_{1}}, s t_{r_{2}} \in$ pmsp $_{t_{2}}$ and $t_{1} \neq t_{2}$, if it is not the case that pmsp $_{t_{1}}$ waits-for pmsp $_{t_{2}}$ and if $s t_{r_{1}}$ is a local step, then a step swap of $s t_{r_{1}} s t_{r_{2}}$ is an equivalent step swap. 
Theorem 1 (Quantized Atomicity) For all paths $p$ there exists an observably equivalent quantized path $p^{\prime}$.

Theorem 2 (Data Race Freedom) For all paths, no two different tasks can access a field of an object in consecutive steps, where at least one of the two accesses changes the value of the field.

Theorem 3 (Mutual Exclusion over Tasks) It can never be the case that two tasks $t_{1}$ and $t_{2}$ overlap execution in a consecutive sequence of steps st $t_{r_{1}} \ldots$ st $_{r_{n}}$ in a path, and in those steps both $t_{1}$ and $t_{2}$ write the same object o, or one reads while the other writes the same object.

\section{Discussion and Related Work}

Implementation We have implemented a prototype of Coqa, called CoqaJava. Polyglot NCM03 was used to construct a translator from CoqaJava to Java. All language features introduced in Fig. 3 are included in the prototype. The implementation dynamically enforces the object capture, freeing, and mutual exclusion semantics of Coqa. Refer to [Lu07] for more details about CoqaJava. The compiler translates CoqaJava to Java. This approach serves as a proof of concept; it unavoidably suffers additional overhead because it is implemented directly on top of Java. For example, every object capture operation in CoqaJava requires a method invocation to realize it in the translated code. The overhead brought by those method invocations can be huge when a large number of capture operations are involved. Even with this highly inefficient implementation, preliminary benchmark results in [Lu07] show that CoqaJava programs on single-core CPUs have slowdowns of "only" 20\% - 60\% compared with a Java implementation of the same problem, a result we consider good given the opportunities available for improving it. Making our language more expressive and its implementation more efficient is an important future goal. For instance, we can build a more efficient CoqaJava by building object capture into the lower level Virtual Machine. It will also be interesting to add more concurrency-related language features, such as futures and synchronization constraints. Optimization techniques should also be able to minimize the amount of capture information that needs to be retained at runtime since many objects are completely local.

Deadlocks. Deadlock will be a more common occurrence in Coqa: accessing shared objects without using subtasking can potentially produce deadlocks. A primary task of writing and debugging Coqa programs will be refactoring code into the correct quanta to both minimize sharing and avoid deadlock. While these extra deadlocks may make it sound like a step backward has been taken, there is reason to be optimistic: Coqa programs will inherently have fewer semantically distinct interleavings, and thus the probability of catching deadlocks before deployment will be significantly greater since there will a much greater likelihood of exercising the different interleaving cases during program testing. 
There are two forms of deadlock arising in Coqa. The first is inherent in twophase locking, when an object is read captured by two tasks but neither task can further write capture it. The second form is cyclically dependent deadlock. The first form of deadlock can be avoided by declaring the class to be exclusive (see Sec. 3). Programmers can also explicitly introduce interleaving via $\Rightarrow$ to break deadlock. There are also many static and dynamic analysis techniques and tools to ensure deadlock freedom; for an overview, see Sin89. Deadlock detection is an important topic of future work for Coqa. We are interested in applying some Java-based analysis tools such Java PathFinder [PF] directly to the target Java code generated by the compiler. The precision of static techniques are reduced due to the combinatorial explosion of interleaving, but Coqa code inherently has many fewer interleavings to consider and so stronger analysis results will de facto be obtained. We are also investigating language design approaches to write deadlock-free programs. It is known that by organizing objects into runtime hierarchies BLR02], deadlock can be effectively avoided; one system we are considering adapting for this purpose is our Pedigree Types [Liu07].

Blocking vs. Rollback. Rollback is a suitable solution in an open database system where the inputs are arbitrary and unknown at the start, and thus a general purpose lock-based deadlock avoidance technique is not possible. Software applications on the other hand are largely closed systems in the sense that the code of an application is often entirely available at deployment time, and so all the code in all the potentially contending threads is known. Therefore, analyzing applications for deadlocks is a more realistic approach in a programming language than in a database system.

Atomicity is commonly addressed in STM systems via rollbacks; example approaches include Harris and Fraser [HF03, Transactional Monitors WJH04 for Java, and Atomos $\left.\mathrm{CMC}^{+} 06\right]$. Compared with blocking systems like ours, STM systems have the appeal of not introducing deadlocks. However, there is a counterpart to deadlock in STM systems, livelock, where rollbacks resulting from contention might result in further contentions and further rollbacks, etc. How frequently livelocks occur is typically gauged by experimental methods. In addition, rollback also may not be as easy as simply discarding the read/write set and retrying (see AbortHandler, etc. in $\left[\mathrm{CMC}^{+} 06\right]$ and onAbort etc. methods in $\mathrm{NMAT}^{+}$07). In terms of performance there have been no detailed studies that we know of comparing locking and rollback. A good overview of the pros and cons of blocking and rollback appears in WHJ06.

Another reason why Coqa does not take a rollback approach is a desire for ubiquitous atomicity, even for I/O-intensive applications. Existing STM systems provide atomicity guarantees only for code explicitly specified by programmers, say, by declaring a block to be atomic; I/O cannot occur in these regions since it cannot generally be undone. In order to make sure that the system can roll back to the state before an abandoned transaction, a STM system needs to perform bookkeeping on the initial state of every transaction. So programmers have to be stingy in the number of atomic blocks declared, to avoid the overhead of such bookkeeping growing unexpectedly large with increasing number 
of threads and transaction sizes. As a result, in a large number of STM systems HF03 WJH04Cra05], code by default runs in a mode with no atomicity guarantees, and the interleaving of this code with atomicity-preserving code in fact can break the atomicity of the latter, an unfortunate consequence known as weak atomicity $\left.\mathrm{CMC}^{+} 06\right]$.

Atomicity in Actors and Other Languages. Our work is most related to Actor languages. Actors Agh90 AMST97 provide a simple concurrent model where each actor is a concurrent unit. Inter-actor communication is only via asynchronous messaging. Ubiquitous atomicity is preserved in the Actor model because executing each actor method does not depend on the state of other actors and so each method execution is trivially serializable. However, the Actor model's per-method atomicity is only a local property in the sense that it neither includes more than one actor nor other methods invoked by the current method. So, Coqa is a significant extension to the Actor notion of atomicity. Morevoer, Actors are a model more suited to loosely-coupled distributed programming: for tightly-coupled message sequences, programming them in the pure Actor model means breaking off each method after each send and wrapping up the continuation as a new actor method. Typically when Actor languages are implemented Arm96 Mil HO06 YBS86, additional language constructs (such as futures, and explicit continuation capture) are included to ease programmability, but there is still a gap in that the most natural mode of programming, synchronous messaging, is not fully supported, only limited forms thereof. We elect to support full synchronous messaging so that Coqa coding style can be extremely close to standard programming practice.

Argus Lis88 pioneered the study of atomicity in object-oriented languages. Like actors it is focused on loosely coupled computations in a distributed context, so it is quite remote in purpose from Coqa but there is still overlap in some dimensions. Argus allows nested transactions, called subactions. Unlike our subtasking, when a subaction ends, all its objects are merged with the parent action, instead of being released early to promote parallelism as a subtask does. Guava [BST00 was designed with the same philosophy as Coqa: code is concurrencyaware by default. The property Guava enforces is race freedom, which is a weaker and more low-level property than the quantized atomicity of Coqa.

\section{Conclusion and Future Work}

Coqa is a foundational study of how concurrency can be built deeply into object models; our particular target is tightly coupled computations running concurrently on multi-core CPUs. Coqa has a very simple and sound foundation - it is defined via only three forms of messaging, which account for (normal) local message send, thread spawning via asynchronous message send, and atomic subtasking via synchronous nonlocal send. We formalized Coqa as the language KernelCoqa, and proved that it observes a wide range of good concurrency properties, in particular quantized atomicity. We justify our approach by implementing CoqaJava, a Java extension incorporating all of the Coqa features. 


\section{References}

[Agh90] Agha, G.: ACTORS: A model of Concurrent computations in Distributed Systems. MITP, Cambridge, Mass (1990)

[AMST97] Agha, G., Mason, I.A., Smith, S.F., Talcott, C.L.: A foundation for actor computation. Journal of Functional Programming 7(1), 1-72 (1997)

[Arm96] Armstrong, J.: Erlang — a Survey of the Language and its Industrial Applications. In: INAP 1996 - The 9th Exhibitions and Symposium on Industrial Applications of Prolog, Hino, Tokyo, Japan, pp. 16-18 (1996)

[BLR02] Boyapati, C., Lee, R., Rinard, M.: Ownership types for safe programming: preventing data races and deadlocks. In: OOPSLA 2002, Seattle, Washington, USA, pp. 211-230. ACM Press, New York, NY, USA (2002)

[BST00] Bacon, D.F., Strom, R.E., Tarafdar, A.: Guava: a dialect of java without data races. In: OOPSLA 2000, pp. 382-400. ACM Press, New York (2000)

$\left[\mathrm{CMC}^{+}\right.$06] CarlStrom, B., McDonald, A., Chafi, H., Chung, J., Minh, C., Kozyrakis, C., Olukotun, K.: The atomos transactional programming language. In: PLDI 2006, Ottawa, Ontario, Canada (June 2006)

[Cra05] Cray Inc. Chapel Specification (2005)

[Gra78] Gray, J.: Notes on data base operating systems. In: Flynn, M.J., Jones, A.K., Opderbeck, H., Randell, B., Wiehle, H.R., Gray, J.N., Lagally, K., Popek, G.J., Saltzer, J.H. (eds.) Operating Systems. LNCS, vol. 60, pp. 393-481. Springer, Heidelberg (1978)

[HF03] Harris, T., Fraser, K.: Language support for lightweight transactions. In: OOPSLA 2003, pp. 388-402 (2003)

[HO06] Haller, P., Odersky, M.: Event-based programming without inversion of control. In: Dumke, R.R., Abran, A. (eds.) IWSM 2000. LNCS, vol. 2006, Springer, Heidelberg (2001)

[Lip75] Lipton, R.J.: Reduction: a method of proving properties of parallel programs. Commun. ACM 18(12), 717-721 (1975)

[Lis88] Liskov, B.: Distributed programming in argus. Commun. ACM 31(3), 300$312(1988)$

[Liu07] Liu, Y.D.: Interaction-Oriented Programming, PhD thesis, Johns Hopkins University, Baltimore, MD, USA, (2007), electronic copy available at http://www.cs.jhu.edu/ yliu/thesis/

[Lom77] Lomet, D.B.: Process structuring, synchronization, and recovery using atomic actions. SIGOPS Oper. Syst. Rev. 11(2), 128-137 (1977)

[LS89] Lamport, L., Schneider, F.B.: Pretending atomicity. Technical Report TR89-1005, Digital Equipment Corporation (1989)

[Lu07] Lu, X.: Coqa: A Concurrent Programming Model with Ubiquitous Atomicity. PhD thesis, Johns Hopkins University, Baltimore, MD, USA (November 2007), electronic copy available at http://www.cs.jhu.edu/ xiaoqilu/thesis/

[Mil] Miller, M.: The E Language, http://www.erights.org

[MPA05] Manson, J., Pugh, W., Adve, S.V.: The java memory model. In: POPL 2005, pp. 378-391. ACM Press, New York (2005)

[NCM03] Nystrom, N., Clarkson, M.R., Myers, A.C.: Polyglot: An extensible compiler framework for java. In: Hedin, G. (ed.) CC 2003. LNCS, vol. 2622, pp. 138-152. Springer, Heidelberg (2003) 
[NMAT ${ }^{+}$07] Ni, Y., Menon, V., Adl-Tabatabai, A.-R., Hosking, A.L., Hudson, R.L., Moss, J.E.B., Saha, B., Shpeisman, T.: Open nesting in software transactional memory. In: ACM SIGPLAN 2007 Symposium on Principles and Practice of Parallel Programming (March 2007)

[PF] Java PathFinder at, http://javapathfinder.sourceforge.net/

[Sin89] Singhal, M.: Deadlock detection in distributed systems. IEEE Computer 22(11), 37-48 (1989)

[WHJ06] Welc, A., Hosking, A.L., Jagannathan, S.: Transparently reconciling transactions with locking for java synchronization. In: Thomas, D. (ed.) ECOOP 2006. LNCS, vol. 4067, pp. 148-173. Springer, Heidelberg (2006)

[WJH04] Welc, A., Jagannathan, S., Hosking, A.L.: Transactional monitors for concurrent objects. In: Odersky, M. (ed.) ECOOP 2004. LNCS, vol. 3086, pp. 519-542. Springer, Heidelberg (2004)

[YBS86] Yonezawa, A., Briot, J.-P., Shibayama, E.: Object-oriented concurrent programming abcl/1. In: OOPLSA 1986: Conference proceedings on Objectoriented programming systems, languages and applications, pp. 258-268. ACM Press, New York (1986) 